\section{ORIGINAL RESEARCH}

S.W. Hetts

J.D. English

C.F. Dowd

R.T. Higashida

J.T. Scanlon

V.V. Halbach

\title{
Pediatric Intracranial Aneurysms: New and Enlarging Aneurysms after Index Aneurysm Treatment or Observation
}

\begin{abstract}
BACKGROUND AND PURPOSE: Children with brain aneurysms may be at higher risk than adults to develop new or enlarging aneurysms in a relatively short time. We sought to identify comorbidities and angiographic features in children that predict new aneurysm formation or enlargement of untreated aneurysms.
\end{abstract}

MATERIALS AND METHODS: Retrospective analysis of the University of California-San Francisco Pediatric Aneurysm Cohort data base including medical records and imaging studies was performed.

RESULTS: Of 83 patients harboring 114 intracranial aneurysms not associated with brain arteriovenous malformations or intracranial arteriovenous fistulas, 9 (8.4\%) developed new or enlarging brain aneurysms an average of 4.2 years after initial presentation. Comorbidities that may be related to aneurysm formation were significantly higher in patients who developed new aneurysms (89\%) as opposed to patients who did not develop new or enlarging aneurysms (41\%; RR, 9.5; 95\% Cl, $1.9 \%-48 \% ; P=$ .0099). Patients with multiple aneurysms at initial presentation were more likely than patients with a single aneurysm at presentation to develop a new or enlarging aneurysm (RR, $6.2 ; 95 \% \mathrm{Cl}, 2.1 \%-185$; $P=.0058$ ). Patients who initially presented with at least 1 fusiform aneurysm were more likely to develop a new or enlarging aneurysm than patients who did not present with a fusiform aneurysm (RR, $22 ; 95 \% \mathrm{Cl}, 3.6 \%-68 \% ; P=.00050)$. Index aneurysm treatment with parent artery occlusion also was associated with higher risk of new aneurysm formation (RR, $4.2 ; 95 \% \mathrm{Cl}, 1.3 \%-13 \% ; P=.024)$. New aneurysms did not necessarily arise near index aneurysms. The only fatality in the series was due to subarachnoid hemorrhage from a new posterior circulation aneurysm arising 20 months after index anterior circulation aneurysm treatment in an immunosuppressed patient.

CONCLUSIONS: Patients who presented with a fusiform aneurysm had a significantly greater incidence of developing a new aneurysm or enlargement of an index aneurysm than did those who presented with a saccular aneurysm. In our patient cohort, 8 of the 9 children who eventually developed new or enlarging brain aneurysms initially presented with fusiform aneurysm morphology. Other comorbidities or multiple aneurysms were also common in these patients at initial presentation.

ABBREVIATIONS: $\mathrm{ACA}=$ anterior cerebral artery; $\mathrm{AcomA}=$ anterior communicating artery; $\mathrm{Cl}=$ confidence interval; MOPD II = microcephalic osteodysplastic primordial dwarfism type II; PCA = posterior cerebral artery; PcomA = posterior communicating artery; $R R=$ risk ratio; $S C I D=$ severe combined immunodeficiency; VBJ = vertebrobasilar junction

B rain aneurysms are rare in children, representing only approximately $1 \%$ of all intracranial aneurysms. ${ }^{1-4}$ Previous reports have suggested that the location, morphology, presentation, and etiology of brain aneurysms differ in children compared with adults. Although some of the risk factors classically associated with aneurysm formation and rupture are usually absent in the pediatric population (eg, cigarette smoking and chronic hypertension), other risk factors (eg, collagen vascular diseases) may be more common in children developing aneurysms. Aneurysmal vasculopathies may affect certain vulner-

Received December 29, 2010; accepted after revision March 21, 2011.

From the Departments of Radiology (S.W.H., J.D.E., C.F.D., R.T.H., J.T.S., V.V.H.) and Neurology (J.D.E.), University of California-San Francisco, San Francisco, California.

Portions of the data presented in this manuscript previously presented at: ASNR Annual Meeting, May 10, 2010, Boston, Massachusetts; other portions were previously presented at: WNRS Annual Meeting, September 30, 2010, Wailea, Hawaii.

Please address correspondence to Steven W. Hetts, MD, Division of Interventional Neuroradiology, Department of Radiology, UCSF Medical Center, 505 Parnassus Ave, L-352, San Francisco, CA 94143-0628; e-mail: steven.hetts@radiology.ucsf.edu

三E Indicates article with supplemental on-line tables.

http://dx.doi.org/10.3174/ajnr.A2680 able arterial segments, or there may be factors that have a field effect on the entire arterial tree. ${ }^{5}$

We previously described the cohort of all pediatric patients treated for intracranial aneurysms at our institution in the last 3 decades. ${ }^{6}$ The present study is a more detailed subset analysis of those patients who developed new or enlarging brain aneurysms during clinical and angiographic follow-up. The purpose of the current study was to identify comorbidities that may be related to aneurysm formation as well as other prospective risk factors for de novo aneurysm formation or enlargement such as number of aneurysms at initial presentation, morphology of the index aneurysm, nature of initial clinical presentation, and method of initial aneurysm treatment.

\section{Materials and Methods}

Under an institutional review board-approved protocol, retrospective analysis of medical records and imaging studies between 1981 and 2010 was performed. Patients age 18 years or younger at the time of initial diagnosis of intracranial aneurysm (regardless of symptoms at presentation) were included. Patients with coexistent AVM or arteriovenous fistula were excluded. As we have described previously, ${ }^{6}$ 

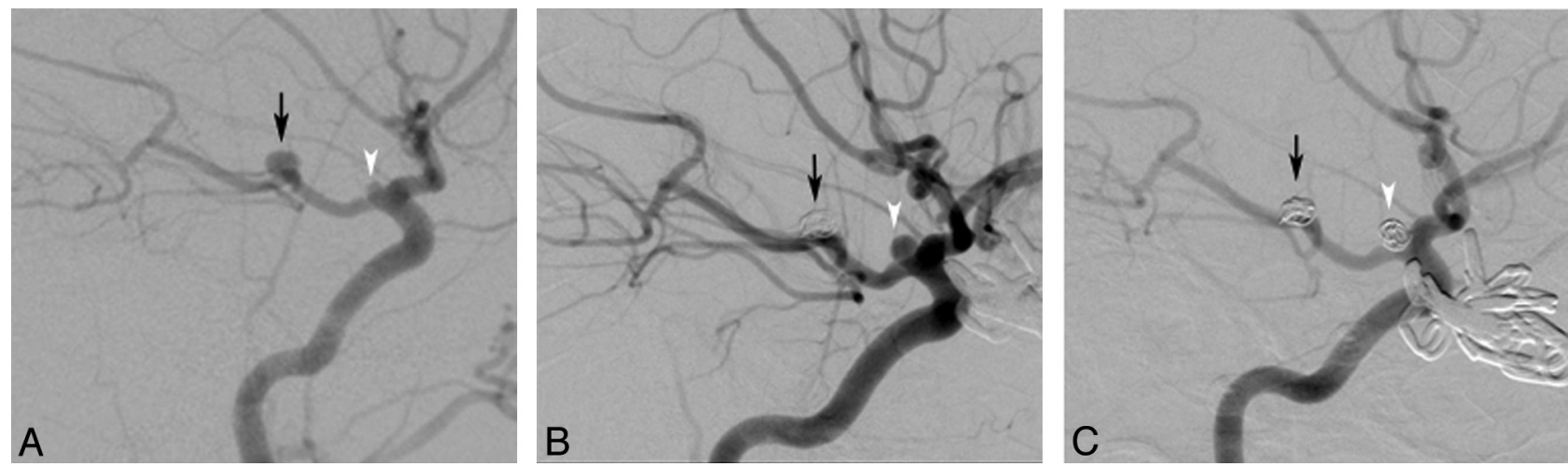

Fig 1. Multiple saccular intracranial aneurysms with enlargement. A 17-year-old female adolescent with MOPD II (patient 9 from On-line Tables 1 and 2) presented with SAH, was found to have 10 saccular intracranial aneurysms, and underwent surgical clipping of multiple left anterior circulation aneurysms (not shown). The lateral projection of a right ICA catheter angiogram $(A)$ demonstrates filling of a basilar tip aneurysm (black arrow) across the PcomA as well as filling of a smaller aneurysm at the anterior origin of the PcomA (white arrowhead). The basilar tip aneurysm underwent selective endovascular coiling at the time of initial SAH and remained well occluded on 9-month asymptomatic surveillance angiography (black arrow B). The previously identified right PcomA aneurysm, however, had increased in size from $1 \mathrm{~mm}$ in diameter to $2.5 \mathrm{~mm}$ in diameter (white arrowhead, $A$ versus $B$ ) and was also treated with selective endovascular coiling (white arrowhead, C).

comorbidities defined as possibly related to aneurysm formation included trauma, cerebral ischemia, vasculopathy, dermatologic syndrome, hormonal syndrome, hematologic disorder, immunologic syndrome, cardiovascular malformation, neoplasm, epilepsy, and other known genetic syndromes or family history thereof. Presence of these conditions was determined from patient medical chart and imaging review. Statistical analyses (2-tailed $t$ tests for continuous variables and Fisher exact test for ordinal variables) comparing patients who developed new or enlarging brain aneurysms to those who did not develop new or enlarging aneurysms were performed by using StataSE version 10.0 (Stata, College Station, Texas).

\section{Results}

Of 83 patients harboring 114 intracranial aneurysms not associated with brain AVMs or intracranial arteriovenous fistulas, $9(8.4 \%)$ developed new or enlarging brain aneurysms an average of 4.2 years after initial presentation (On-line Tables $1-3)$. Whereas the mean duration of follow-up in patients developing new or enlarging aneurysms was 5.9 years, the mean follow-up duration in patients not found to have new or enlarging aneurysms was only 2.6 years (On-line Table 3 ). Demographic characteristics such as sex and age at initial presentation were similar in patients who developed new or enlarging aneurysms and in those who did not.

Comorbidities that may be related to aneurysm formation were significantly higher in patients who developed new aneurysms $(89 \%)$ as opposed to patients who did not develop new or enlarging aneurysms (41\%; RR, 9.5; 95\% CI, 1.9\%-48\%; $P=.0099$; On-line Table 3). Comorbid conditions found in $>1$ patient in the total patient cohort ${ }^{6}$ included trauma, immune dysfunction, cardiovascular malformation, dermatologic disorder, hormonal abnormality, cerebral ischemia, and genetic syndromes. In the total cohort 1 patient each had a hematologic disorder, epilepsy, neoplasia, or systemic vasculopathy. Specific comorbidities found in patients developing new or enlarging aneurysms (On-line Tables 1 and 2) included AIDS, X-linked SCID, tricuspid atresia, vascular birthmark, MOPD II, hemiatrophy, and trauma (2 patients). Factoring out cases of trauma, which ideally should be monophasic events, patients developing new or enlarging aneurysms were still more likely to harbor a nontraumatic comorbidity than patients who did not develop new or enlarging aneurysms (RR, 4.4; 95\% CI, 1.3\%-15\%; $P=.024$ ).

Patients with multiple intracranial aneurysms at initial presentation were more likely than patients with a single aneurysm at presentation to develop a new or enlarging aneurysm (RR, 6.2; 95\% CI, 2.1\%-18\%; $P=.0058$; On-line Table 3). Among patients developing new or enlarging aneurysms (Online Tables 1 and 2), 4 patients had 1 aneurysm at initial presentation, 2 patients had 2 aneurysms at initial presentation, 2 patients had 3 aneurysms at initial presentation, and 1 patient with MOPD II had 10 saccular aneurysms at initial presentation (Fig 1).

Index aneurysm morphology and mode of treatment also seemed to correlate with forming or growing aneurysms. Patients who initially presented with at least 1 fusiform aneurysm (On-line Table 3) were more likely to develop a new or enlarging aneurysm than patients who did not present with a fusiform aneurysm (RR, 22; 95\% CI, 3.6\%-68\%; $P=.00050)$. Of patients who went on to develop new or enlarging aneurysms, $89 \%$ had a fusiform index aneurysm; $89 \%$ of second or enlarging aneurysms were either fusiform aneurysms or focally enlarging components of an underlying fusiform aneurysm (Online Tables 1 and 2). Index aneurysm treatment with parent artery occlusion (endovascular or surgical) was associated with higher risk of new aneurysm formation or aneurysm enlargement (RR, 4.2; 95\% CI, 1.3\%-13\%; $P=.024$ ) than selective clipping, coiling, or observation with parent artery preservation (On-line Table 3 ). Five patients $(1,3,6,7$, and 8 from On-line Tables 1 and 2) had parent artery occlusion as their first therapy. New or enlarging aneurysms arose in the distribution of the occluded artery in 2 patients ( 1 and 7 from Online Tables 1 and 2) and in nonadjacent locations in 3 patients (3, 6, and 8 from On-line Tables 1 and 2).

Although new aneurysms sometimes arose in locations distant from the treated index aneurysm, some patients developed new aneurysms in arterial segments adjacent to previously treated aneurysms (On-line Tables 1 and 2 and Figs 2 and 3). Having at least 1 posterior circulation aneurysm at initial presentation, however, was not statistically different $(P=.69)$ between patients who did not go on to develop new or enlarging aneurysms (24\%) and those who did (33\%). 


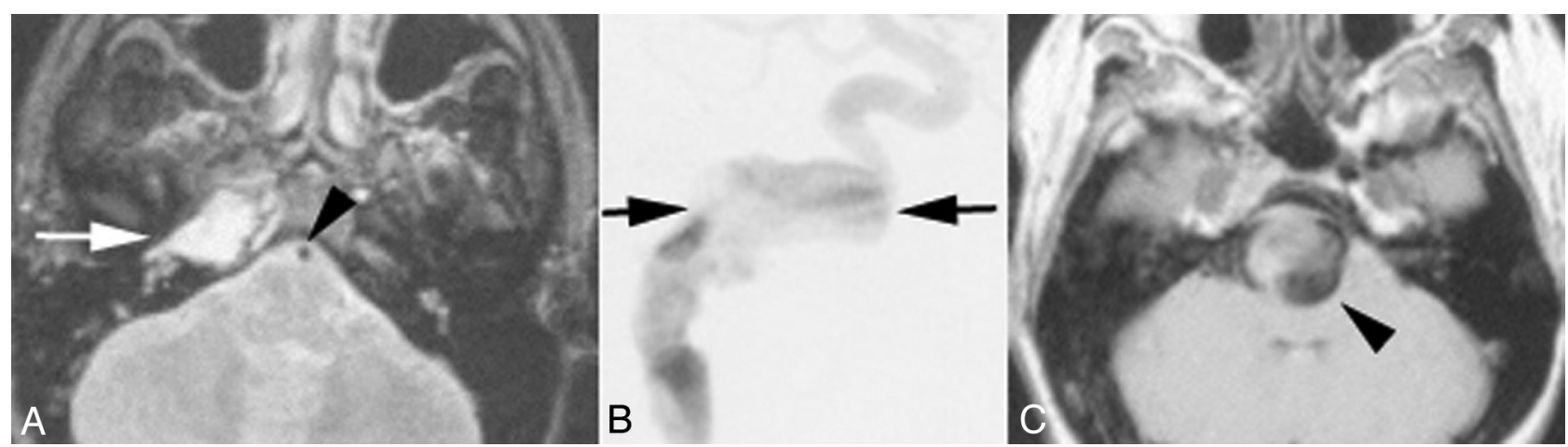

Fig 2. De novo aneurysm formation in a segment remote from the site of initial aneurysm treatment (remote segment vasculopathy). A 15-year-old female adolescent with history of remote head trauma years earlier (patient 8 from On-line Table 1) presented with pulsatile tinnitus and a vascular mass behind the right tympanic membrane. T2-weighted MR imaging (A) demonstrated a hyperintense mass (white arrow) in the right petrous bone; note that the basilar artery flow void (black arrowhead) in the prepontine cistern is normal in caliber. A right ICA angiogram in the lateral (B) projection confirms that the right petrous mass (black arrows) is a large fusiform aneurysm of the cervicopectoral ICA. The ICA was occluded with detachable balloons and the patient's tinnitus resolved. Six years later, the patient re-presented with diplopia and swallowing difficulties concerning for cranial neuropathies. $T 1$-weighted axial $(C)$ MR imaging performed at that time demonstrated a new mass (black arrowhead) centered in the prepontine cistern compressing the pons. The mass was confirmed on left vertebral artery angiography (not shown) to be a new giant fusiform aneurysm of the vertebrobasilar artery junction.

Clinical presentation at the time of index aneurysm diagnosis differed between those patients who did not subsequently develop new or enlarging aneurysms and those who did (On-line Table 3). Specifically, although $11 \%$ of patients who did not go on to develop new or enlarging aneurysms were asymptomatic at initial presentation (eg, aneurysm discovered on neuroimaging performed for aneurysm screening or for a nonvascular indication), $44 \%$ of patients who eventually did develop new or enlarging aneurysms were asymptomatic at the time of index aneurysm diagnosis. Not surprisingly, $67 \%$ of patients developing a new or enlarging aneurysm had that second or enlarging aneurysm identified in the asymptomatic state. One patient who developed a new vertebrobasilar junction aneurysm 6 years after index ICA aneurysm occlusion re-presented with symptoms of brain stem mass effect including diplopia and lower cranial neuropathies (Fig 2). Another patient who developed a new vertebrobasilar junction aneurysm 3 years after index ICA aneurysm occlusion re-presented with neck pain. The only fatality in the series was due to SAH from a de novo posterior circulation aneurysm arising 20 months after index anterior circulation aneurysm treatment in a patient with AIDS.

\section{Discussion}

With regard to brain aneurysms, children are not small adults. The formation of new and enlarging cerebral aneurysms in adults is infrequent, but we report a high rate of new or enlarging untreated aneurysms in pediatric patients: $8.4 \%$ during a mean follow-up of just 5.9 years. In a study of adult patients imaged with 3T MRA 5 years after brain aneurysm coiling, $1.54 \%$ had de novo aneurysms. ${ }^{7}$ In a study of adult patients having undergone ICA occlusion for aneurysm treatment, $3 \mathrm{~T}$ MRA at a mean of 50.3 months after takedown (range, 0-107 months), showed no de novo aneurysms. ${ }^{8}$ Our current study relies on catheter angiographic imaging follow-up, which may be more sensitive than 3T MRA for small aneurysms. Apart from our patient with MOPD II and numerous small saccular intracranial aneurysms treated with both clips and coils, however, the new and enlarging aneurysms identified in our series probably would be detectable by the MRA methods used in the screening of adult patients. Thus, it seems likely that pediatric patients develop new and enlarging aneurysms at a faster pace than their adult counterparts. The probable inconstancy of aneurysm growth rates, ${ }^{9}$ as underscored by patient 1 (Fig 3), makes it challenging to develop a protocol for aneurysm imaging surveillance that would be applicable to all pediatric patients.

A significant bias in our current study is that follow-up of patients developing new or enlarging aneurysms was significantly longer ( $5.9 \pm 4.2$ versus $2.9 \pm 3.9$ years; $P=.02$ ) than in those not found to have new or enlarging aneurysms. Once a patient is lost to follow-up, it is unknown if he or she subsequently develops a new or enlarging aneurysm. Thus, our study may actually underestimate the number of children with cerebral aneurysms who go on to develop additional or enlarging untreated aneurysms during their lifetimes. Given that the life expectancy of children is long, losing these patients to follow-up is of significant clinical concern.

Many of the risk factors associated with aneurysm enlargement in adults are not typically present in children, including cigarette smoking, chronic hypertension, and excessive alcohol consumption. ${ }^{10,11}$ Larger aneurysms have been reported to have greater likelihood of growing than smaller aneurysms, possibly because they have a greater area of wall vulnerable to growth or because it is easier to measure growth in a large aneurysm than in a small aneurysm near the limit of resolution of noninvasive imaging techniques often used for serial measurement. ${ }^{12}$ In our series, index aneurysms in patients who eventually developed new or enlarging aneurysms were not significantly larger than those in patients who did not go on to develop new or enlarging aneurysms (On-line Table 3). With regard to de novo aneurysm formation, however, it is not clear that having a larger index aneurysm per se would connote a greater risk of second aneurysm formation unless the largeness of the first aneurysm is taken to suggest an underlying extensive vasculopathy that affects multiple portions of the cerebral vasculature. ${ }^{5}$ The significant enlargement of small saccular aneurysms in patient 9 of this series (Fig 1) also underscores the point that even small aneurysms can enlarge quickly. Multiplicity of aneurysms at initial presentation (On-line Table 3) also seems to be a risk factor for formation of new or enlarge- 

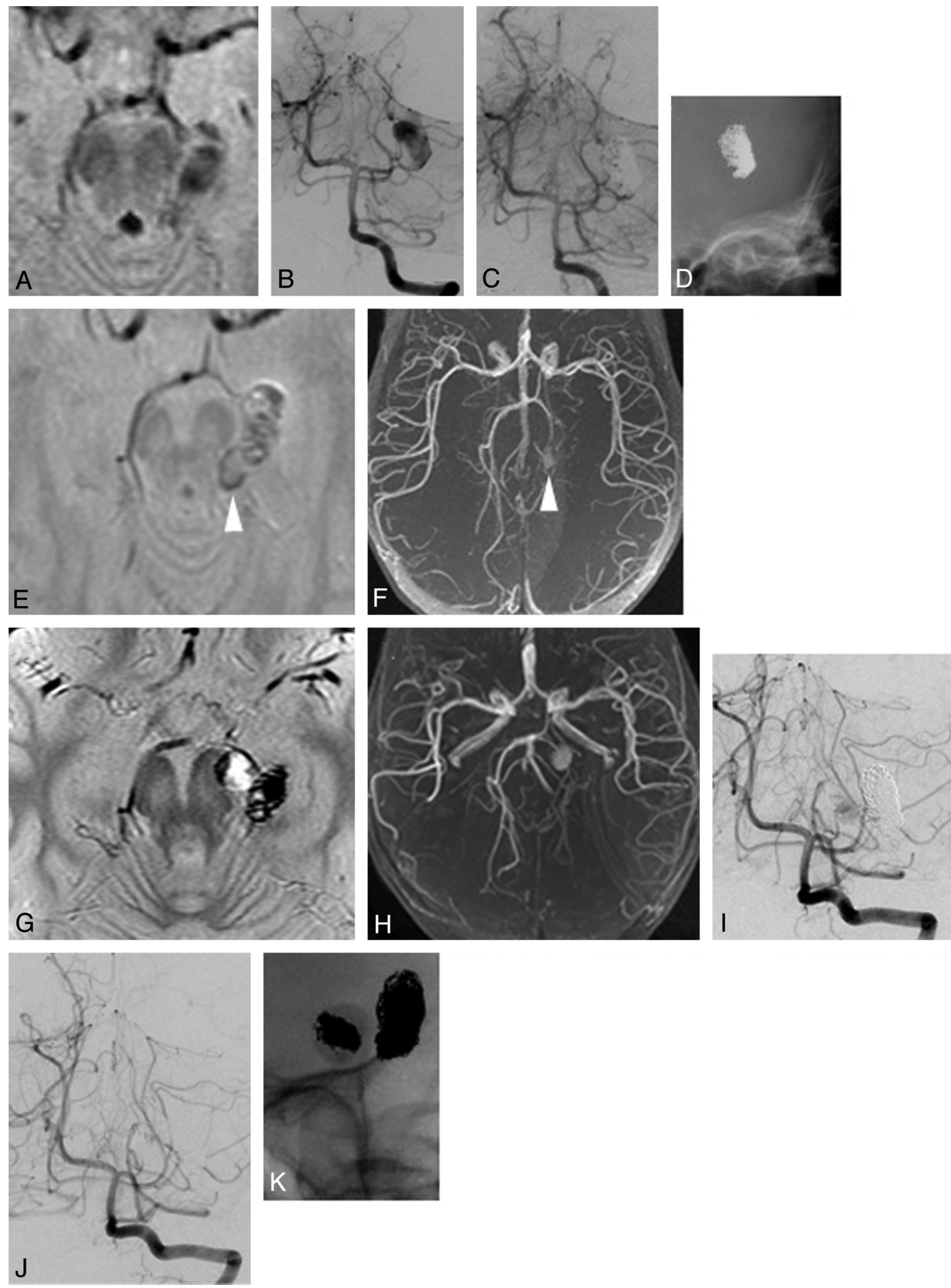

Fig 3. De novo aneurysm formation in a segment adjacent to a previously treated aneurysm (adjacent segment vasculopathy). A 3-year-old boy who fell from standing and hit his head (patient 1 from On-line Tables 1 and 2) was found on MR imaging to have a mass centered in the left ambient cistern (A). This mass was identified as a fusiform aneurysm of the left P2/3 PCA on conventional angiography (B). Endovascular coil occlusion of the aneurysm ( $C$ and $D)$ was performed, resulting in parent artery occlusion of the PCA at and distal to the aneurysm. The patient developed no clinical deficit, and diffusion-weighted MR imaging (not shown) confirmed no infarct related to PCA occlusion in this young patient. Surveillance MR imaging $(E)$ and MRA $(A) 7$ months later demonstrated no change in the ambient cistern coil mass and no patent aneurysm; a small amount of thrombus in the posterior portion of the aneurysm shines through on the time-of-flight MRA (white arrowheads, $E$ and $A$. Surveillance MR imaging $(G)$ and MRA $(H) 4.5$ years after initial aneurysm treatment, however, demonstrated a new mass compressing the left cerebral peduncle $(G)$. This was confirmed on conventional angiography ( $)$ to be a de novo aneurysm of the left P2 PCA just medial to the previously treated aneurysm. Selective endovascular coiling of the new aneurysm was performed $(U)$, and a postcoiling unsubtracted $x$-ray image $(K)$ confirms that the newly placed coils do not abut the previous coil mass, itself unchanged since initial therapy $(D)$. 
ment of existing untreated aneurysms in the children in our study.

Several comorbidities have been reported previously to correlate with increased risk of brain aneurysm formation in adults and children: medial or internal elastic membrane defects, ${ }^{13}$ mutations in genes affecting maintenance of the extracellular matrix, ${ }^{14,15}$ and congenital syndromes such as posterior fossa malformations, hemangiomas, arterial anomalies, coarctation of the aorta and other cardiac defects, eye abnormalities, sternal clefting and/or a supraumbilical raphe (PHACES); tuberous sclerosis (PHACES); neurofibromatosis (PHACES); and Marfan syndrome. Ehlers-Danlos syndrome type IV (the vascular type or Sack-Barabas syndrome) results from autosomal dominant mutations in the gene for type III procollagen (COL3A1) and leads to sudden transverse or longitudinal arterial tears (dissections) followed by pseudoaneurysms, walled-in hematomas, and ruptures with only occasional documentation of pre-existing aneurysms. ${ }^{16-18}$ Loss of function mutations in the pericentrin gene result in clinical MOPD II (as in patient 9 of our series), that in turn results not only in dwarfism but also in cerebrovascular malformations involving weak-walled arteries, including both aneurysms and Moyamoya disease. ${ }^{19,20}$ Presumably other as yet undetermined genetic factors play a role in various familial aneurysm syndromes that typically present in adulthood. The identification of comorbidities at a higher frequency in patients who went on to develop new or enlarging aneurysms bespeaks the importance of detailed medical history taking in all aneurysm patients. Presence of such comorbid conditions may argue in favor of more frequent noninvasive surveillance imaging in selected patients.

The role of trauma in our series was interesting. Two of the 3 patients (patients 1 and 6) in the de novo and enlarging aneurysm subgroup presented with mild acute trauma (fall from standing and fall from a tree, respectively). Thus, these aneurysms might not be mechanistically due to trauma but instead have been discovered incidentally on the imaging performed to evaluate potential acute head injury. This leaves only patient 8 , who suffered skull base fractures during a motor vehicle crash years before presentation with a large cervicopectoral ICA aneurysm. Delayed pseudoaneurysm formation following traumatic arterial dissection reminds us that trauma is not a monophasic event; in fact, trauma's lingering effects on the vasculature can be profound. The fact that patient 8 had such an extensive cervicopectoral aneurysm may suggest that she had a predisposing arterial wall vulnerability that caused what was probably initially a small ICA injury (because she did not have vascular imaging at the time of the motor vehicle crash, this can only be speculated) to progress into a large fusiform aneurysm remodeling her petrous carotid canal (Fig 2). Patient 8's de novo aneurysm at the vertebrobasilar junction may have arisen due to increased posterior circulation flow after ICA occlusion, with that increased flow affecting an artery already made vulnerable either by prior trauma or by an undetected vasculopathy, though the latter seems more likely given the normal caliber of the basilar artery at the time of index ICA aneurysm treatment.

If a dissecting aneurysm is defined as "aneurysmal dilation of the artery caused by the presence of blood within its wall," 21 it is often challenging to determine which aneurysms are due to an arterial tear with concomitant intramural hemorrhage. Unless a dissection flap or nearby luminal stenosis (due to compression of the true lumen by thrombus in the false lumen or arterial wall) are apparent on angiography, then fusiform luminal dilation cannot be definitely ascribed to a dissecting etiology. In our series, patient 1 (Fig $3 A,-B)$ harbors a fusiform index aneurysm of the PCA — where the artery lies adjacent to the tentorium - that is classically described as dissecting in nature, as such lesions are often identified in the setting of minor trauma. The index fusiform cervicopectoral ICA aneurysm of patient 2 (Fig $2 A-C$ ) might similarly be attributed to vascular dissection in the setting of prior significant trauma; some investigators also would consider this patient's de novo vertebrobasilar junction aneurysm to be dissecting based on its morphology and location (Fig $2 D-G) .{ }^{21}$ Given the inherent limitations of angiography, however, high-resolution MR imaging may be able to differentiate intramural from intraluminal thrombus ${ }^{22}$ and thus may better identify the dissecting nature of aneurysms.

Fusiform morphology of intracranial aneurysms not only presents treatment challenges but, in our cohort, also seems to indicate a higher propensity for additional aneurysm formation or enlargement. It has been posited that many morphologically fusiform aneurysms represent dissecting aneurysms in which thrombus is present between layers of the arterial wall in addition to intraluminal thrombus forming at sites of slow flow in the dilated arterial lumen. ${ }^{23}$ Patient 1 of our series demonstrates a fusiform aneurysm morphology with either intraluminal or mural thrombus in the distal portion of a PCA aneurysm that persisted after successful occlusion of the more proximal portion of the aneurysm (thus, the parent PCA). The development of a new PCA aneurysm proximally adjacent to but apparently separate from the initially treated aneurysm raises 2 possibilities. First, the segment of the PCA vulnerable to aneurysm formation extended proximal to the location of the initial aneurysm but that vulnerable segment was not dilated at the time of index aneurysm treatment. Second, the first coiling was incomplete due to intraluminal or mural thrombus extending proximal to the site of coiling and that clot later lysed revealing a more proximal lobule of the index aneurysm. The latter possibility, however, is very unlikely given serial MR imaging examinations (Fig 3) that demonstrate no extension of the index aneurysm to the cerebral peduncle initially or at first follow-up (with normal-appearing P2 PCA segments at those time points), but the more delayed follow-up imaging showed a new P2 PCA aneurysm impinging on the cerebral peduncle. New steady-state free precession MR imaging techniques, ${ }^{22}$ by allowing direct visualization of aneurysm inner and outer walls, may allow better evaluation of aneurysms with associated intraluminal or intramural thrombus. Application of such advanced MR imaging techniques also may begin to address the possibility of mural vasculopathy extending proximal or distal to an angiographically evident aneurysm, allowing stratification of patients into those who may need closer follow-up surveillance or potentially more extensive parent artery occlusion therapy.

\section{Conclusions}

Patients who presented with a fusiform aneurysm had a significantly greater incidence of developing a new aneurysm or 
enlargement of an index aneurysm than did those who presented with a saccular aneurysm. In our patient cohort, 8 of the 9 children who eventually developed new or enlarging brain aneurysms initially presented with fusiform aneurysm morphology. Other comorbidities or multiple aneurysms were also common in these patients at initial presentation.

\section{Acknowledgments}

We thank Michael T. Lawton, MD; Nalin Gupta, MD, PhD; and Heather Fullerton, MD, for collaborative clinical care. We also thank S. Claiborne Johnston, $\mathrm{MD}, \mathrm{PhD}$, for advice on statistical matters and Jared Narvid, MD, for assistance with data base organization.

Disclosures: Christopher F. Dowd, Consultant: NeuroVasx, Other Financial Interests: Boston Scientific, Details: Clinical Trial Adjudicator.

\section{References}

1. Gerosa M, Licata C, Fiore DL, et al. Intracranial aneurysms of childhood. Childs Brain 1980;6:295-302

2. Meyer FB, Sundt TM, Jr, Fode NC, et al. Cerebral aneurysms in childhood and adolescence. J Neurosurg 1989;70:420-25

3. Ostergaard JR, Voldby B. Intracranial arterial aneurysms in children and adolescents. J Neurosurg 1983;58:832-37

4. Locksley HB, Sahs AL, Sandler R. Report on the cooperative study of intracranial aneurysms and subarachnoid hemorrhage. 3. Subarachnoid hemorrhage unrelated to intracranial aneurysm and A-V malformation. A study of associated diseases and prognosis. J Neurosurg 1966;24:1034-56

5. Lasjaunias PL. Segmental identity and vulnerability in cerebral arteries. Interv Neuroradiol 2000;30:113-24

6. Hetts SW, Narvid J, Sanai N, et al. Intracranial aneurysms in childhood: 27year single-institution experience. AJNR Am J Neuroradiol 2009;30:1315-24

7. Sprengers ME, van Rooij WJ, Sluzewski M, et al. MR angiography follow-up 5 years after coiling: frequency of new aneurysms and enlargement of untreated aneurysms. AJNR Am J Neuroradiol 2009;30:303-07

8. de Gast AN, Sprengers ME, van Rooij WJ, et al. Long-term 3T MR angiography follow-up after therapeutic occlusion of the internal carotid artery to detect possible de novo aneurysm formation. AJNR Am J Neuroradiol 2007;28:508-10

9. Koffijberg H, Buskens E, Algra A, et al. Growth rates of intracranial aneurysms: exploring constancy. J Neurosurg 2008;109:176-85

10. Juvela S, Porras M, Poussa K. Natural history of unruptured intracranial aneurysms: probability of and risk factors for aneurysm rupture. J Neurosurg 2000;93:379-87

11. So TY, Dowling R, Mitchell PJ, et al. Risk of growth in unruptured intracranial aneurysms: a retrospective analysis. J Clin Neurosci 2010;17:29-33

12. Burns JD, Huston J, 3rd, Layton KF, et al. Intracranial aneurysm enlargement on serial magnetic resonance angiography: frequency and risk factors. Stroke 2009;40:406-11

13. Ostergaard JR, Reske-Nielsen E, Buhl J. Deficiency of reticular fibers in cerebral arteries. On the etiology of saccular aneurysms in childhood. Br J Neurosurg 1989;3:113-15

14. Ruigrok YM, Rinkel GJ, Wijmenga C. Genetics of intracranial aneurysms. Lancet Neurol 2005;4:179-89

15. Ruigrok YM, Rinkel GJ, van't Slot R, et al. Evidence in favor of the contribution of genes involved in the maintenance of the extracellular matrix of the arterial wall to the development of intracranial aneurysms. Hum Mol Genet 2006; 15:3361-68

16. Barabas AP. Ehlers-Danlos syndrome. In: Greenhalgh RM, Mannick JA, eds. The Cause and Management of Aneurysms. London: W.B. Saunders; 1990:57-67

17. Pepin M, Schwarze U, Superti-Furga A, et al. Clinical and genetic features of Ehlers-Danlos syndrome type IV, the vascular type. $N$ Engl $\mathrm{J} \mathrm{Med}$ 2000;342:673-80

18. Barabas AP. Ehlers-Danlos syndrome type IV. N Engl J Med 2000;343:366, author reply 368

19. Waldron J, Hetts S, Armstrong-Wells J, et al. Multiple intracranial aneurysms and Moyamoya in patients with Majewski's osteodysplastic primordial dwarfism type II. J Neurosurg Pediatr 2009;4:439-44

20. Bober MB, Khan N, Kaplan J, et al. Majewski osteodysplastic primordial dwarfism type II (MOPD II): expanding the vascular phenotype. Am J Med Genet 2010;152A:960-65

21. Massimi L, Moret J, Tamburrini G, et al. Dissecting giant vertebro-basilar aneurysms. Childs Nerv Syst 2003;19:204-10

22. Martin AJ, Hetts SW, Dillon WP, et al. MR imaging of partially thrombosed cerebral aneurysms: characteristics and evolution. AJNR Am J Neuroradiol 2011;32:346-51

23. Lasjaunias $\mathrm{P}$, Wuppalapati $\mathrm{S}$, Alvarez $\mathrm{H}$, et al. Intracranial aneurysms in children aged under 15 years: review of 59 consecutive children with 75 aneurysms. Childs Nerv Syst 2005;21:437-50 